\title{
A Similarity Invariant and the Commutant of some Multiplication Operators on the Sobolev Disk Algebra
}

\author{
Ruifang Zhao \\ Department of Mathematics, East China University of Science and Technology, Shanghai 200237, China \\ Correspondence should be addressed to Ruifang Zhao,rfzhao@ecust.edu.cn \\ Received 31 March 2012; Accepted 19 July 2012 \\ Academic Editor: Gideon Schechtman \\ Copyright (c) 2012 Ruifang Zhao. This is an open access article distributed under the Creative \\ Commons Attribution License, which permits unrestricted use, distribution, and reproduction in \\ any medium, provided the original work is properly cited. \\ Let $R(\mathbb{D})$ be the algebra generated in Sobolev space $W^{22}(\mathbb{D})$ by the rational functions with poles \\ outside the unit disk $\overline{\mathbb{D}}$. In this paper, we study the similarity invariant of the multiplication \\ operators $M_{g}$ in $\mathcal{L}\left(R(\mathbb{D})\right.$ ), when $g$ is univalent analytic on $\mathbb{D}$ or $M_{g}$ is strongly irreducible. And \\ the commutants of multiplication operators whose symbols are composite functions, univalent \\ analytic functions, or entire functions are studied.
}

\section{Introduction}

Let $\Omega$ be an analytic Cauchy domain in the complex plane and let $W^{22}(\Omega)$ denote the Sobolev space,

$$
W^{22}(\Omega)=\left\{f \in L^{2}(\Omega, d A): \begin{array}{l}
\text { the distributional partial derivatives of first } \\
\text { and second order of } f \text { belong to } L^{2}(\Omega, d A)
\end{array}\right\}
$$

$d A$ denotes the planar Lebesgue measure. For $f, g \in W^{22}(\Omega)$, we define

$$
\langle f, g\rangle=\sum_{|\alpha| \leq 2} \int D^{\alpha} f \overline{D^{\alpha} g} d A .
$$


Then $W^{22}(\Omega)$ is a Hilbert space and a Banach algebra with identity under an equivalent norm. $W^{22}(\Omega)$ can be continuously embedded in the space $C(\bar{\Omega})$ of continuous functions on $\bar{\Omega}$ by Sobolev embedding theorem.

Let $R(\Omega)$ be the subalgebra generated by the rational functions with poles outside $\bar{\Omega}$. When $\Omega=\mathbb{D}$, the unit disc, we call $R(\mathbb{D})$ Sobolev disk algebra. For $f \in R(\mathbb{D})$, the multiplication operator $M_{f}$ on $R(\mathbb{D})$ is defined by $M_{f}(g)=f g, g \in R(\mathbb{D})$. Then $R(\mathbb{D})=$ $\mathscr{A}\left(M_{z}\right) e_{0}$, where $e_{0}$ is the identity in $R(\mathbb{D})$ and $\mathscr{A}\left(M_{z}\right)$ is the algebra generated by $M_{z}$ and identity. In fact, $R(\mathbb{D})$ consists of all analytic functions in $W^{22}(\mathbb{D})$. We have the following properties of the space $R(\mathbb{D})$ and the multiplication operators on it.

Proposition 1.1 (see [1]). (i) Hilbert space $R(\mathbb{D})$ has an orthogonal basis $\left\{e_{n}\right\}_{n=0}^{+\infty}$, where

$$
e_{n}=\beta_{n} z^{n}, \quad \beta_{n}=\left[\frac{n+1}{\left(3 n^{4}-n^{2}+2 n+1\right) \pi}\right]^{1 / 2}, \quad n=0,1,2, \ldots
$$

(ii) As a functional Hilbert space, $R(\mathbb{D})$ has reproducing kernel which is

$$
k(u, v)=\sum_{n=0}^{+\infty} \beta_{n}^{2} u^{n} \bar{v}^{n}
$$

Then for $z_{0} \in \overline{\mathbb{D}}$,

$$
k_{z_{0}}=\sum_{n=0}^{\infty}\left(\beta_{n}^{2} \bar{z}_{0}^{n}\right) z^{n}
$$

(iii) If $f(z)=\sum_{n=0}^{+\infty} f_{n} z^{n}$ is analytic on $\mathbb{D}$, then $f(z) \in R(\mathbb{D})$ if and only if $\sum_{n=0}^{+\infty}\left|f_{n}\right|^{2} / \beta_{n}^{2}<$ $+\infty$.

(iv) The operator $M_{f}$ admits the following matrix representation with respect to $\left\{e_{n}\right\}_{n=0}^{+\infty}$ :

$$
M_{f}=\left(\begin{array}{cccccc}
c_{0} & & & & \\
c_{1} \frac{\beta_{0}}{\beta_{1}} & c_{0} & & 0 & \\
c_{2} \frac{\beta_{0}}{\beta_{2}} & c_{1} \frac{\beta_{1}}{\beta_{2}} & c_{0} & & \\
c_{3} \frac{\beta_{0}}{\beta_{3}} & c_{2} \frac{\beta_{1}}{\beta_{3}} & c_{1} \frac{\beta_{2}}{\beta_{3}} & c_{0} & \\
\vdots & \vdots & \ddots & \ddots & \ddots
\end{array}\right) .
$$

Note that $R(\mathbb{D})$ is a subset of the disk algebra $A(\mathbb{D})$, hence a subset of $H^{\infty}$. Because of the special definition of the inner product and the complex behavior of the boundary value, the structure of the space $R(\mathbb{D})$ is much more complicated than $H^{\infty}$ or $H^{2}$. For more details about the Sobolev disk algebra, the reader refers to [1-3]. 
Let $\mathscr{\ell}$ be a complex separable Hilbert space and $\mathcal{L}(\mathscr{l})$ denote the collection of bounded linear operators on $\mathscr{\ell}$. One of the basic problems in operator theory is to determine when two operators $A$ and $B$ in $\mathcal{L}(\mathscr{L})$ are similar. A quantity (quantities) or a property (properties) $P$ is similarity invariant (invariants) if $A$ has $P$ and $A \sim B$ implies that $B$ has $P$ [2]. From this point of view, one of the basic problems in operator theory mentioned above is to determine the similarity invariants. There have already been a lot of results on the similarity invariants of operators, especially that of nonadjoint operators, which can be found in, for example, [4-6]. In [7], Wang et al. proved that in $R(\mathbb{D}), M_{f}$ is similar to $M_{z^{n}}$ if and only if $f$ is an $n$-Blaschke product. In this paper, we study the similarity invariant of the multiplication operators $M_{g}$ in $\mathcal{L}(R(\mathbb{D}))$, when $g$ is univalent analytic on $\mathbb{D}$ or $M_{g}$ is strongly irreducible.

It is well known that the commutant of a bounded linear operator or operators on a complex, separable Hilbert space plays an important role in determining the structure of this operator or these operators. The commutant of a multiplication operator on Sobolev disk algebra has been studied in the literature (see [1-3]). In this paper, we describe the commutant of the multiplication operator $M_{f g}$ when $g$ is an $n$-Blaschke product. And by this result, we generalize the result which is obtained by Liu and Wang in [3]. Moreover, we study the commutants of the multiplication operators whose symbols are composite functions, univalent analytic functions, or entire functions.

\section{Similarity Invariant of Some Multiplication Operators}

In this section, we will characterize the similarity invariant of some multiplication operators on Sobolev disk algebra. Here, we briefly recall some background information.

For $T$ in $\mathcal{L}(\mathscr{\ell})$, let $\sigma(T), \sigma_{p}(T)$, and $\sigma_{e}(T)$ be the spectrum, point spectrum, and essential spectrum of a bounded linearly operator $T$, respectively. An operator $A$ in $\mathcal{L}(\mathscr{\ell})$ is said to be a Cowen-Douglas operator with index $n$ if there exists $\Omega$, a connected open subset of complex plane $\mathbb{C}$, and $n$, a positive integer, such that

(i) $\Omega \subset \sigma(A)=\{\lambda \in \mathbb{C}: A-\lambda$ is not invertible $\}$;

(ii) $\operatorname{ran}(A-\lambda):=\{y$; $(A-\lambda) x=y, x \in \mathscr{\ell}\}=\mathscr{H}$ for $\lambda$ in $\Omega$;

(iii) $\operatorname{nul}(A-\lambda):=\operatorname{dim} \operatorname{ker}(A-\lambda)=n$ for $\lambda$ in $\Omega$;

(iv) $\bigvee\{\operatorname{ker}(A-\lambda): \lambda \in \Omega\}=\mathscr{\ell}$,

where (iv) is equivalent to (iv)' ([8]);

(iv)' there exists $\lambda_{0}$ in $\Omega$, such that $\bigvee\left\{\operatorname{ker}\left(A-\lambda_{0}\right)^{k}: k \geq 1\right\}=\mathscr{H}$.

$B_{n}(\Omega)$ denotes the collection of Cowen-Douglas operators with index $n$.

For $T \in \mathcal{L}(\mathscr{\ell})$, the set of operators which commute with it is $\mathscr{A}^{\prime}(T)$. That is $\mathscr{A}^{\prime}(T)=$ $\{A \in \mathcal{L}(\mathscr{L}): A T=T A\}$. Operator $T$ is strongly irreducible if there is no nontrivial idempotent in $\mathcal{A}^{\prime}(T)[8,9]$. Denote $(S I)$ the set of all strongly irreducible operators on $\mathscr{d}$.

Definition 2.1. Let $\mathscr{\ell}$ be a Hilbert space and $A, B$ be in $\mathcal{L}(\mathscr{\ell})$. $\tau_{A B}$ is said to be a Rosenblum operator on $\mathcal{L}(\mathscr{\ell})$ if for arbitrary $C \in \mathcal{L}(\mathscr{L}), \tau_{A B}(C)=A C-C B$.

Lemma 2.2 (see $[1])$. Let $f$ be in $R(\mathbb{D})$, then

(i) $\sigma\left(M_{f}\right)=f(\overline{\mathbb{D}})$; 
(ii) $\sigma_{e}\left(M_{f}\right)=f(\partial \mathbb{D})$, where $\partial \mathbb{D}$ denotes the boundary of the unit disc $\mathbb{D}$;

(iii) let $z_{0} \in \mathbb{D}$ and $f\left(z_{0}\right) \notin f(\partial \mathbb{D})$. Denote the component of $\rho_{s-F}\left(M_{f}\right)$ containing $f\left(z_{0}\right)$ as $\Omega$, then $M_{f}^{*} \in \mathbb{B}_{n}(\Omega)$, where $n$ is the number of the zeros of $f(z)-f\left(z_{0}\right)$ in $\mathbb{D}$.

Lemma 2.3 (see $[10])$. Set $f \in R(\mathbb{D})$ and $B_{a}(z)=(z-a) /(1-\bar{a} z), a \in \mathbb{D}$. Then $M_{f \circ B_{a}} \sim M_{f}$.

Theorem 2.4. Let $f$ and $g$ be in $R(\mathbb{D})$ and be univalent and analytic on $\mathbb{D}$. Then $M_{f} \sim M_{g}$ if and only if $f(\mathbb{D})=g(\mathbb{D})$.

Proof. " $\Rightarrow$ ": Set $M_{f} \sim M_{g}$. By Lemma 2.2, we have

$$
f(\overline{\mathbb{D}})=\sigma\left(M_{f}\right)=\sigma\left(M_{g}\right)=g(\overline{\mathbb{D}}), \quad f(\mathbb{T})=\sigma_{e}\left(M_{f}\right)=\sigma_{e}\left(M_{g}\right)=g(\mathbb{T}),
$$

where $\mathbb{T}$ is the unit circle. Since $f$ and $g$ are univalent and analytic on $\mathbb{D}$, then

$$
f(\mathbb{T})=\partial f(\overline{\mathbb{D}})=\partial g(\overline{\mathbb{D}})=g(\mathbb{T}) .
$$

Therefore,

$$
f(\mathbb{D})=f(\overline{\mathbb{D}}) \backslash f(\mathbb{T})=g(\overline{\mathbb{D}}) \backslash g(\mathbb{T})=g(\mathbb{D}) .
$$

" $\Leftarrow$ ": Set $f(\mathbb{D})=g(\mathbb{D})=\Omega$. Because $g$ is univalent analytic from $\mathbb{D}$ to $\Omega, g^{-1}: \Omega \rightarrow \mathbb{D}$ is also univalent analytic. Then $g^{-1} \circ f$ is injective and surjective analytic function on $\mathbb{D}$. If $g^{-1} \circ f\left(z_{0}\right)=0$, there exists a Möbius transform $\varphi$ with $\varphi_{z_{0}}=\left(z-z_{0}\right) /\left(1-\overline{z_{0}} z\right)$ and a complex number $c$ with $|c|=1$ such that $g^{-1} \circ f(z)=c \varphi_{z_{0}}$ (see [11]). Therefore $f(z)=g\left(c \varphi_{z_{0}}(z)\right)$. By Lemma 2.3, $M_{f} \sim M_{g}$.

Lemma 2.5 (see [3]). Given $f \in R(\mathbb{D})$, the following are equivalent:

(i) $M_{f}^{*} \in B_{1}(\Omega)$;

(ii) $\mathcal{A}^{\prime}\left(M_{f}\right)=\left\{M_{g}: g \in R(\mathbb{D})\right\}$;

(iii) $M_{f} \in(S I)$.

Theorem 2.6. Let $f, g \in R(\mathbb{D})$ and $f$ is univalent analytic on $\mathbb{D}$. $M_{f} \sim M_{g}$ if and only if there exists a function $X=c\left(\left(z-z_{0}\right) /\left(1-\overline{z_{0}} z\right)\right)$ such that $f=g \circ X$, where $z_{0} \in \mathbb{D}$ and $c \in \mathbb{C},|c|=1$.

Proof. " $\Rightarrow$ ": Suppose that $g$ is not univalent on $\mathbb{D}$. There exists some $\lambda \in g(\mathbb{D})$ such that the number of zeros of $g(z)-\lambda$ on $\mathbb{D}$ is $n>1$. By Lemma 2.2, $M_{g}^{*} \in B_{n}(\Omega)$ where $\Omega$ is a connected open subset of $g(\mathbb{D})$. Since $M_{f} \sim M_{g}$, we have $M_{f}^{*} \in B_{n}(\Omega)$. This contradicts to $M_{f}$ that is a strongly irreducible operator (see Lemma 2.5). So $g$ is univalent analytic on $\mathbb{D}$. By the proof of Theorem 2.4, there exists a function $x=c\left(\left(z-z_{0}\right) /\left(1-\overline{z_{0}} z\right)\right)$ such that $f=g \circ x$, where $z_{0} \in \mathbb{D}$ and $c \in \mathbb{C},|c|=1$.

" $\Leftarrow$ ": By the conditions of the theorem, $g$ is univalent analytic on $\mathbb{D}$. Since $f(\mathbb{D})=$ $g \circ X(\mathbb{D})=g(\mathbb{D})$, we have $M_{f} \sim M_{g}$ by Theorem 2.4.

For any operator $T$ on Hilbert space $\mathscr{H}$ and any integer $n, 1 \leq n \leq \infty$, let $T^{(n)}$ denote the direct sum of $n$ copies of $T$ on $\mathscr{H}^{(n)}=\mathscr{H} \oplus \cdots \oplus \mathscr{H}$. 
Lemma 2.7 (see [2]). Let $A_{1}, A_{2}$ be strongly irreducible Cowen-Douglas operators. Assume that $A_{1} \uparrow A_{2}$ and $T=A_{1}^{\left(n_{1}\right)} \oplus A_{2}^{\left(n_{2}\right)}$, where $n_{1}, n_{2}$ are natural numbers. Then for each maximal ideal 2 of $\mathcal{A}^{\prime}(T), 2$ must be one of the following two forms:

$$
\begin{aligned}
& \text { (i) } \partial=\left(\begin{array}{cc}
\partial_{11} & \operatorname{ker} \tau_{A_{1}^{\left(n_{1}\right)}, A_{2}^{\left(n_{2}\right)}} \\
\operatorname{ker} \tau_{A_{2}^{\left(n_{2}\right)}, A_{1}^{\left(n_{1}\right)}} & \mathcal{A}^{\prime}\left(A_{2}^{\left(n_{2}\right)}\right)
\end{array}\right) \text {; } \\
& \text { (ii) } \partial=\left(\begin{array}{cc}
\mathcal{A}^{\prime}\left(A_{1}^{\left(n_{1}\right)}\right) & \operatorname{ker} \tau_{A_{1}^{\left(n_{1}\right)}, A_{2}^{\left(n_{2}\right)}} \\
\operatorname{ker} \tau_{A_{2}^{\left(n_{2}\right)}, A_{1}^{\left(n_{1}\right)}} & \partial_{22}
\end{array}\right) \text {, }
\end{aligned}
$$

where $\partial_{i i}$ is a maximal ideal of $\mathcal{A}^{\prime}\left(A_{i}^{\left(n_{i}\right)}\right), i=1,2$.

Theorem 2.8. Let $f, g \in R(\mathbb{D})$ and $M_{f}, M_{g} \in(S I)$. The following statements are equivalent:

(i) $M_{f} \sim M_{g}$;

(ii) there exist $X_{1}, X_{2}, \ldots, X_{n} \in \operatorname{ker} \tau_{M_{f}, M_{g}}$ and $Y_{1}, Y_{2}, \ldots, Y_{n} \in \operatorname{ker} \tau_{M_{g}, M_{f}}$ such that

$$
X_{1} Y_{1}+X_{2} Y_{2}+\cdots+X_{n} Y_{n}=i d_{R(\mathbb{D})}, \quad Y_{1} X_{1}+Y_{2} X_{2}+\cdots+Y_{n} X_{n}=i d_{R(\mathbb{D})},
$$

where $i d_{R(\mathbb{D})}$ denotes the identity operator of $R(\mathbb{D})$.

Proof. (i) $\Rightarrow$ (ii) : Let $M_{f} \sim M_{g}$. Set $X$ be invertible in $\mathcal{L}(R(\mathbb{D}))$ and $M_{f} X=X M_{g}$. Then $X$ and $X^{-1}$ are what we want.

(ii) $\Rightarrow$ (i) : Since $M_{f}$ and $M_{g}$ are in $(S I)$, we have $M_{f}^{*}$ and $M_{g}^{*}$ that are strongly irreducible and in $B_{1}(\Omega)$ by Lemma 2.5. Computations show

$$
\mathscr{A}^{\prime}\left(M_{f}^{*} \oplus M_{g}^{*}\right)=\left(\begin{array}{cc}
\mathscr{A}^{\prime}\left(M_{f}^{*}\right) & \operatorname{ker} \tau_{M_{f}^{*}, M_{g}^{*}} \\
\operatorname{ker} \tau_{M_{g}^{*}, M_{f}^{*}} & \mathcal{A}^{\prime}\left(M_{g}^{*}\right)
\end{array}\right) .
$$

Suppose that $M_{f}^{*} \nsucc M_{g}^{*}$. By Lemma 2.7, each maximal ideal 2 of $\mathscr{A}^{\prime}\left(M_{f}^{*} \oplus M_{g}^{*}\right)$ must be one of the following two forms

$$
\partial=\left(\begin{array}{cc}
\partial_{11} & \operatorname{ker} \tau_{M_{f}^{*}, M_{g}^{*}} \\
\operatorname{ker} \tau_{M_{g}^{*}, M_{f}^{*}} & \mathscr{A}^{\prime}\left(M_{g}^{*}\right)
\end{array}\right) \quad \text { or } \quad \partial=\left(\begin{array}{cc}
\mathcal{A}^{\prime}\left(M_{f}^{*}\right) & \operatorname{ker} \tau_{M_{f}^{*}, M_{g}^{*}} \\
\operatorname{ker} \tau_{M_{g}^{*}, M_{f}^{*}} & \partial_{22}
\end{array}\right),
$$

where $\partial_{11}$ and $\partial_{22}$ are the maximal ideals of $\mathcal{A}^{\prime}\left(M_{f}^{*}\right)$ and $\mathcal{A}^{\prime}\left(M_{\mathcal{g}}^{*}\right)$, respectively. We can assume that 2 admits the first form. Then

$$
\begin{gathered}
\left(\begin{array}{cc}
0 & Y_{i}^{*} \\
X_{i}^{*} & 0
\end{array}\right) \in 2, \quad i=1,2, \ldots, n \\
\left(\begin{array}{cc}
0 & Y_{i}^{*} \\
X_{i}^{*} & 0
\end{array}\right)\left(\begin{array}{cc}
0 & Y_{i}^{*} \\
X_{i}^{*} & 0
\end{array}\right)=\left(\begin{array}{cc}
Y_{i}^{*} X_{i}^{*} & 0 \\
0 & X_{i}^{*} Y_{i}^{*}
\end{array}\right) \in 2 .
\end{gathered}
$$


It follows that

$$
\sum_{i=1}^{n}\left(\begin{array}{cc}
Y_{i}^{*} X_{i}^{*} & 0 \\
0 & X_{i}^{*} Y_{i}^{*}
\end{array}\right)=\left(\begin{array}{cc}
i d_{R(\mathbb{D})} & 0 \\
0 & i d_{R(\mathbb{D})}
\end{array}\right) \in \mathcal{\partial}
$$

This contradicts that 2 is a maximal ideal. So $M_{f}^{*} \sim M_{g}^{*}$ and $M_{f} \sim M_{g}$.

\section{The Commutant Algebra of Multiplication Operator}

In [3], Liu and Wang give the following proposition.

Proposition 3.1. Let $f(z)=z^{n} h(z) \in R(\mathbb{D}), h(z) \neq 0, z \in \overline{\mathbb{D}}$. Then $\mathcal{A}^{\prime}\left(M_{f}\right)=\mathcal{A}^{\prime}\left(M_{z^{n}}\right) \cap \mathcal{A}^{\prime}\left(M_{h}\right)$.

Let $B_{n}(z)=\prod_{k=1}^{n}\left(z-a_{k}\right) /\left(1-\bar{a}_{k} z\right),\left(\left|a_{k}\right|<1, k=1,2, \ldots, n\right)$ be $n$-Blaschke product. Considering $z^{n}$ is a special $n$-Blaschke product, we study the commutant of $M_{f}$ where $f(z)=$ $B_{n}(z) h(z)$. The following theorem is obtained, and by this result, the above proposition is generalized.

Theorem 3.2. Let $f=B_{n} h \in R(\mathbb{D})$ with $h \in R(\mathbb{D})$ where $h$ does not vanish on $\overline{\mathbb{D}}$. If there exists $0 \neq \lambda \in \mathbb{C}$ such that $h-\lambda$ can be divided by each $\left(z-a_{k}\right) /\left(1-\bar{a}_{k} z\right)$, then $\mathcal{A}^{\prime}\left(M_{f}\right)=\mathcal{A}^{\prime}\left(M_{B_{n}}\right) \cap$ $\mathcal{A}^{\prime}\left(M_{h}\right)$.

To prove the above theorem, we need the following lemmas.

Lemma 3.3 (see [7]). Given $g \in R(\mathbb{D}), M_{g} \sim M_{z^{n}}\left(\sim\left(M_{z}\right)^{n}\right)$ if and only if $g$ is an $n$-Blaschke product.

Lemma 3.4 (see [12]). Let $N$ be a nilpotent operator on $H$ and let $X_{0}=\lambda+N, 0 \neq \lambda \in \mathbb{C}$. If $B, A_{0}, A_{1}, \ldots \in B(H)$ satisfy $\left\|A_{k}\right\| \leq M(k=0,1,2, \ldots)$ and $A_{k} X_{0}=X_{0} A_{k-1}+B,(k=1,2,3 \ldots)$, then $A_{0}=A_{1}=A_{2}=\cdots$.

Now we will prove Theorem 3.2.

Proof. From $h \in R(\mathbb{D})$, we have $M_{f}^{*}=M_{B_{n}}^{*} M_{h}^{*}$. Then, from Lemma 3.3, there exists an invertible operator $X \in \mathcal{L}\left(R(\mathbb{D}), \oplus_{1}^{n} R(\mathbb{D})\right)$ such that $X M_{B_{n}}^{*} X^{-1}=\oplus_{1}^{n} M_{z}^{*}$. It follows that

$$
A=X M_{B_{n}^{*}} X^{-1} X M_{h}^{*} X^{-1}=\left(\oplus_{1}^{n} M_{z}^{*}\right) T
$$

where $T=X M_{h}^{*} X^{-1} \in \mathcal{A}^{\prime}\left(\oplus_{1}^{n} M_{z}^{*}\right)$. So we only need to prove

$$
\mathcal{A}^{\prime}(A)=\mathcal{A}^{\prime}\left(\oplus_{1}^{n} M_{z}^{*}\right) \cap \mathcal{A}^{\prime}(T) .
$$

Since

$$
\begin{aligned}
\oplus_{1}^{n} R(\mathbb{D})= & \operatorname{ker}\left(\oplus_{1}^{n} M_{z}^{*}\right) \oplus\left[\operatorname{ker}\left(\oplus_{1}^{n} M_{z}^{*}\right)^{2}-\operatorname{ker}\left(\oplus_{1}^{n} M_{z}^{*}\right)\right] \\
& \oplus\left[\operatorname{ker}\left(\oplus_{1}^{n} M_{z}^{*}\right)^{3}-\operatorname{ker}\left(\oplus_{1}^{n} M_{z}^{*}\right)^{2}\right] \oplus \cdots \oplus\left[\operatorname{ker}\left(\oplus_{1}^{n} M_{z}^{*}\right)^{k}-\operatorname{ker}\left(\oplus_{1}^{n} M_{z}^{*}\right)^{k-1}\right] \oplus \cdots
\end{aligned}
$$


we have

$$
\oplus_{1}^{n} M_{z}^{*}=\left(\begin{array}{ccccc}
0 & \frac{\beta_{0}}{\beta_{1}} I_{n} & 0 & 0 & \cdots \\
0 & 0 & \frac{\beta_{1}}{\beta_{2}} I_{n} & 0 & \cdots \\
0 & 0 & 0 & \frac{\beta_{2}}{\beta_{3}} I_{n} & \cdots \\
\vdots & \vdots & \vdots & \vdots & \ddots
\end{array}\right) \text {, }
$$

where $I_{n}$ is an $n \times n$ identity matrix. Computations show that $G \in \mathscr{A}^{\prime}\left(\oplus_{1}^{n} M_{z}^{*}\right)$ if and only if

$$
G=\left(\begin{array}{ccccccc}
G_{1} & \frac{\beta_{0}}{\beta_{1}} G_{12} & \frac{\beta_{0}}{\beta_{2}} G_{13} & \frac{\beta_{0}}{\beta_{3}} G_{14} & \cdots & \frac{\beta_{0}}{\beta_{k-1}} G_{1, k} & \cdots \\
0 & G_{1} & \frac{\beta_{1}}{\beta_{2}} G_{12} & \frac{\beta_{1}}{\beta_{3}} G_{13} & \cdots & \frac{\beta_{1}}{\beta_{k-1}} G_{1, k-1} & \cdots \\
0 & 0 & G_{1} & \frac{\beta_{2}}{\beta_{3}} G_{12} & \cdots & \frac{\beta_{2}}{\beta_{k-1}} G_{1, k-2} & \cdots \\
\vdots & \vdots & \vdots & \vdots & \ddots & \vdots & \cdots \\
0 & 0 & 0 & 0 & \cdots & \frac{\beta_{k-2}}{\beta_{k-1}} G_{1,2} & \cdots \\
\vdots & \vdots & \vdots & \vdots & \cdots & \vdots & \ddots
\end{array}\right) .
$$

Therefore,

$$
T=\left(\begin{array}{ccccccc}
T_{1} & \frac{\beta_{0}}{\beta_{1}} T_{12} & \frac{\beta_{0}}{\beta_{2}} T_{13} & \frac{\beta_{0}}{\beta_{3}} T_{14} & \cdots & \frac{\beta_{0}}{\beta_{k-1}} T_{1, k} & \cdots \\
0 & T_{1} & \frac{\beta_{1}}{\beta_{2}} T_{12} & \frac{\beta_{1}}{\beta_{3}} T_{13} & \cdots & \frac{\beta_{1}}{\beta_{k-1}} T_{1, k-1} & \cdots \\
0 & 0 & T_{1} & \frac{\beta_{2}}{\beta_{3}} T_{12} & \cdots & \frac{\beta_{2}}{\beta_{k-1}} T_{1, k-2} & \cdots \\
\vdots & \vdots & \vdots & \vdots & \ddots & \vdots & \cdots \\
0 & 0 & 0 & 0 & \cdots & \frac{\beta_{k-2}}{\beta_{k-1}} T_{1,2} & \cdots \\
\vdots & \vdots & \vdots & \vdots & \cdots & \vdots & \ddots
\end{array}\right) .
$$


Set $g_{k}=\left(z-a_{k}\right) /\left(1-\overline{a_{k}} z\right), 1 \leq k \leq n$, and $B_{n}=g_{1} g_{2} \cdots g_{n}$. Then for each $g_{k}$, there exists $\varphi_{k} \in R(\mathbb{D})$ such that $h-\lambda=g_{k} \varphi_{k}, 1 \leq k \leq n$. So

$$
T=X M_{h}^{*} X^{-1}=X M_{g_{k}}^{*} X^{-1} X M_{\varphi_{k}}^{*} X^{-1}+\bar{\lambda} i d_{\oplus_{1}^{n} R(\mathbb{D})}=V_{k} S_{k}+\bar{\lambda} i d_{\oplus_{1}^{n} R(\mathbb{D})},
$$

where $V_{k}=X M_{g_{k}}^{*} X^{-1}, S_{k}=X M_{\varphi_{k}}^{*} X^{-1}$. For $k=1,2,3, \ldots, n, j=1,2,3, \ldots, n, M_{\varphi_{k}}$, and $M_{g_{j}}$ pairwise commute. Hence, $V_{k}, S_{j}$ pairwise commute too. Since $T_{1}=\left.T\right|_{\operatorname{ker}\left(\oplus_{1}^{n} M_{z}^{*}\right)}$ and

$$
\operatorname{ker}\left(\oplus_{1}^{n} M_{z}^{*}\right)=\operatorname{ker} X M_{B_{n}}^{*} X^{-1}=\operatorname{ker} V_{1} V_{2} \cdots V_{n}
$$

for all $x \in \operatorname{ker}\left(\oplus_{1}^{n} M_{z}^{*}\right)$,

$$
\left(T-\bar{\lambda} i d_{\oplus_{1}^{n} R(\mathbb{D})}\right)^{n} x=V_{1} S_{1} V_{2} S_{2} \cdots V_{n} S_{n} x=S_{1} S_{2} \cdots S_{n} V_{1} V_{2} \cdots V_{n} x=0 .
$$

Therefore, $T_{1}-\bar{\lambda} I_{n}$ is nilpotent operator. Now we set $N=T_{1}-\bar{\lambda} I_{n}$, that is $T_{1}=\bar{\lambda} I_{n}+N$. So

$$
\begin{aligned}
& A=\oplus_{1}^{n} M_{z}^{*} T
\end{aligned}
$$

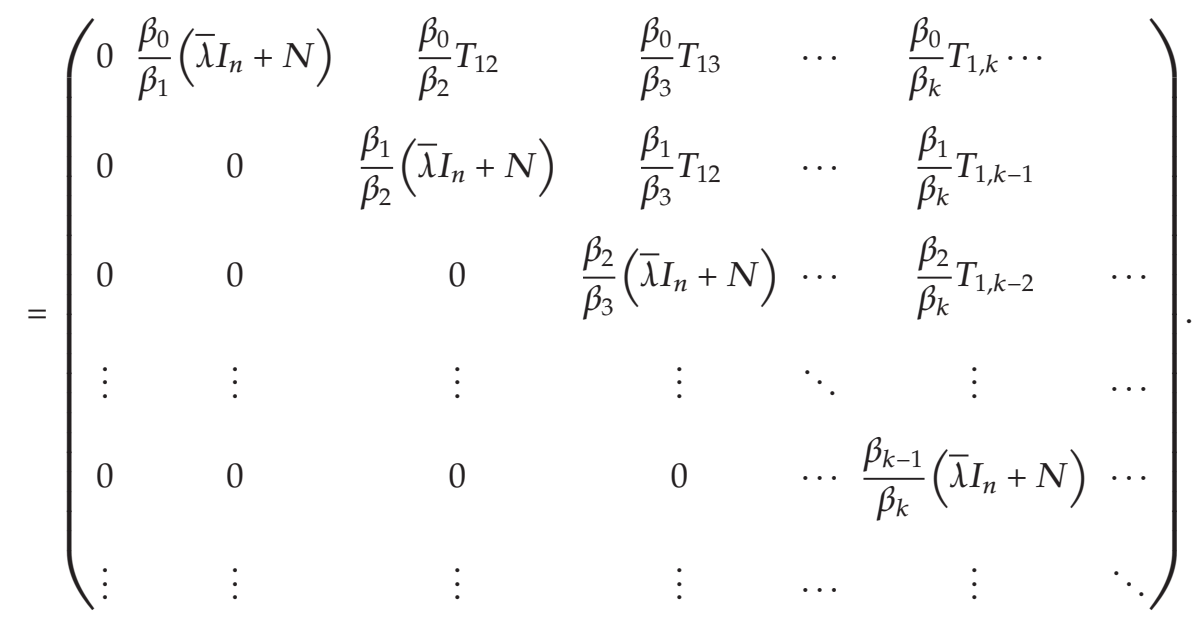

So we only need to prove that $\mathscr{A}^{\prime}(A) \subset \mathcal{A}^{\prime}\left(\oplus_{1}^{n} M_{z}^{*}\right)$. In fact, if $Q \in \mathscr{A}^{\prime}(A) \subset \mathcal{A}^{\prime}\left(\oplus_{1}^{n} M_{z}^{*}\right)$,

$$
\begin{aligned}
Q A=A Q & \Longrightarrow Q T\left(\oplus_{1}^{n} M_{z}^{*}\right)=T\left(\oplus_{1}^{n} M_{z}^{*}\right) Q \\
& \Longrightarrow Q T\left(\oplus_{1}^{n} M_{z}^{*}\right)=T Q\left(\oplus_{1}^{n} M_{z}^{*}\right) \\
& \Longrightarrow(Q T-T Q)\left(\oplus_{1}^{n} M_{z}^{*}\right)=0 \\
& \Longrightarrow\left(\oplus_{1}^{n} M_{z}\right)\left(T^{*} Q^{*}-Q^{*} T^{*}\right)=0
\end{aligned}
$$

It follows from $\operatorname{ker}\left(\oplus_{1}^{n} M_{z}\right)=\{0\}$ that $T^{*} Q^{*}=Q^{*} T^{*}$. Namely, $T Q=Q T$. 
We are now in need to prove that $\mathscr{A}^{\prime}(A) \subset \mathcal{A}^{\prime}\left(\oplus_{1}^{n} M_{z}^{*}\right)$. Suppose that $Q \in \mathcal{A}^{\prime}(A)$. Since $h$ does not vanish on $\overline{\mathbb{D}}, \overline{\operatorname{ran} M_{h}}=R(\mathbb{D})$ and so $\operatorname{ker} T=\{0\}$. Because $A=\left(\oplus_{1}^{n} M_{z}^{*}\right) T=T\left(\oplus_{1}^{n} M_{z}^{*}\right)$,

$$
\operatorname{ker} A=\operatorname{ker}\left(\oplus_{1}^{n} M_{z}^{*}\right), \quad \operatorname{ker} A^{k}=\operatorname{ker}\left(\oplus_{1}^{n} M_{z}^{*}\right)^{k}
$$

It follows from $Q A=A Q$ that $\operatorname{ker} A$ and $\operatorname{ker} A^{k}$ are both the invariant subspaces of $Q$. Since

$$
\oplus_{1}^{n} R(\mathbb{D})=\operatorname{ker} A \oplus\left(\operatorname{ker} A^{2} \ominus \operatorname{ker} A\right) \oplus\left(\operatorname{ker} A^{3} \ominus \operatorname{ker} A^{2}\right) \oplus \cdots \oplus\left(\operatorname{ker} A^{k} \ominus \operatorname{ker} A^{k-1}\right) \oplus \cdots,
$$

$A$ admits the matrix representation (3.5) with the above decomposition. So

$$
Q=\left(\begin{array}{ccccc}
Q_{1} & Q_{12} & Q_{13} & Q_{14} & \cdots \\
0 & Q_{2} & Q_{23} & Q_{24} & \cdots \\
0 & 0 & Q_{3} & Q_{34} & \cdots \\
\vdots & \vdots & \vdots & \vdots & \vdots
\end{array}\right)
$$

From $Q A=A Q$, we have

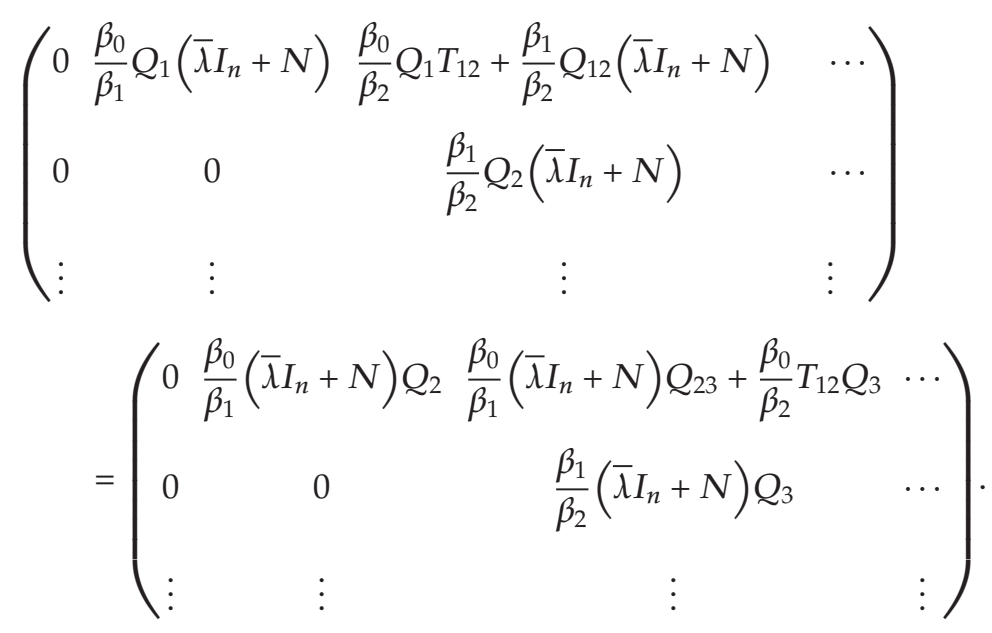

Comparing the $(n, n+1)$ entries of both sides, we have $Q_{1}=Q_{2}=Q_{3}=\cdots$ by Lemma 3.4. Comparing the $(n, n+2)$ entries of $Q A$ and $A Q$, we have

$$
\frac{\beta_{n+1}}{\beta_{n}}\left(\bar{\lambda} I_{n}+N\right) Q_{n+1, n+2}=\frac{\beta_{n}}{\beta_{n-1}} Q_{n, n+1}\left(\bar{\lambda} I_{n}+N\right)+\left(Q_{1} T_{12}-T_{12} Q_{1}\right) .
$$

It follows from Lemma 3.4 that

$$
\frac{\beta_{n+1}}{\beta_{n}} Q_{n+1, n+2}=\frac{\beta_{n}}{\beta_{n-1}} Q_{n, n+1} .
$$


Setting $Q_{12}^{\prime}=\left(\beta_{1} / \beta_{0}\right) Q_{12}$, we have

$$
Q_{n, n+1}=\frac{\beta_{1} \beta_{n-1}}{\beta_{0} \beta_{n}} Q_{12}=\frac{\beta_{n-1}}{\beta_{n}} Q_{12}^{\prime}
$$

Inductively, if $Q_{n, n+k-1}=\left(\beta_{n-1} / \beta_{n+k-2}\right) Q_{1, k}^{\prime}$, where $Q_{1, k}^{\prime}=\left(\beta_{k-1} / \beta_{0}\right) Q_{1 k}$, we need to prove $Q_{n, n+k}=\left(\beta_{n-1} / \beta_{n+k-1}\right) Q_{1, k+1}^{\prime}$. Comparing the $(n, n+k+1)$ entries of $Q A$ and $A Q$, we have

$$
\begin{aligned}
\frac{\beta_{n+k}}{\beta_{n}}\left(\bar{\lambda} I_{n}+N\right) Q_{n+1, n+k+1}= & \frac{\beta_{n+k-1}}{\beta_{n-1}} Q_{n, n+k}\left(\bar{\lambda} I_{n}+N\right)+\left(T_{12} Q_{1, k}^{\prime}-Q_{1, k}^{\prime} T_{12}\right) \\
& +\left(T_{13} Q_{1, k-1}^{\prime}-Q_{1, k-1}^{\prime} T_{13}\right)+\cdots+\left(T_{1, k+1} Q_{1}-Q_{1} T_{1, k+1}\right) .
\end{aligned}
$$

Therefore, by Lemma 3.4,

$$
\frac{\beta_{n+k}}{\beta_{n}} Q_{n+1, n+k+1}=\frac{\beta_{n+k-1}}{\beta_{n-1}} Q_{n, n+k} .
$$

Computations show $Q_{n, n+k}=\left(\beta_{n-1} / \beta_{n+k-1}\right) Q_{1, k+1}^{\prime}$. Since $Q$ is the form of (3.5), $Q \in \mathcal{A}^{\prime}\left(\oplus_{1}^{n} M_{z}^{*}\right)$. So $\mathscr{A}^{\prime}\left(M_{f}\right)=\mathscr{A}^{\prime}\left(M_{B_{n}}\right) \cap \mathscr{A}^{\prime}\left(M_{h}\right)$.

Corollary 3.5. Let $f=B_{n} h \in R(\mathbb{D})$ with $h \in R(\mathbb{D})$ where $h$ does not vanish on $\overline{\mathbb{D}}$. If there exists $0 \neq \lambda \in \mathbb{C}$ that $h-\lambda$ can be divided by $B_{n}$, then $\mathcal{A}^{\prime}\left(M_{f}\right)=\mathcal{A}^{\prime}\left(M_{B_{n}}\right) \cap \mathcal{A}^{\prime}\left(M_{h}\right)$.

By the following lemma, we discuss the commutant of the multiplication operators whose symbols are composite functions in $R(\mathbb{D})$.

Lemma 3.6. For $T$ in $\mathcal{L}(R(\mathbb{D}))$ and $f$ in $R(\mathbb{D})$ the following are equivalent:

(i) $T \in \mathscr{A}^{\prime}\left(M_{f}\right)$;

(ii) for all $\alpha \in \mathbb{D}, T^{*} k_{\alpha} \perp(f-f(\alpha)) R(\mathbb{D})$;

(iii) there is a set $J \subset \mathbb{D}$ such that $\sum_{\alpha \in J}(1-|\alpha|)=\infty$ and for all $\alpha \in J, T^{*} k_{\alpha} \perp(f-f(\alpha)) R(\mathbb{D})$.

Proof. (i) $\Rightarrow$ (ii) Let $T \in \mathscr{A}^{\prime}\left(M_{f}\right)$. For all $g \in R(\mathbb{D})$ and $\alpha \in \mathbb{D}$, we have

$$
\begin{aligned}
\left\langle(f-f(\alpha)) g, T^{*} k_{\alpha}\right\rangle & =\left\langle T M_{f} g, k_{\alpha}>-f(\alpha)<T g, k_{\alpha}\right\rangle \\
& =\left\langle M_{f} T g, k_{\alpha}\right\rangle-f(\alpha)\left\langle T g, k_{\alpha}\right\rangle \\
& =f(\alpha)(T g)(\alpha)-f(\alpha)(T g)(\alpha) \\
& =0 .
\end{aligned}
$$

(ii) $\Rightarrow$ (iii) Let $J=\mathbb{D}$. 
International Journal of Mathematics and Mathematical Sciences

(iii) $\Rightarrow$ (i) Let $T \in \mathcal{L}(R(\mathbb{D}))$ with $T^{*} k_{\alpha} \perp(f-f(\alpha)) R(\mathbb{D})$ for all $\alpha \in J$. For $g \in R(\mathbb{D})$ and $\alpha \in J$, we have

$$
\begin{aligned}
0 & =\left\langle(f-f(\alpha)) g, T^{*} k_{\alpha}\right\rangle \\
& =\left(T M_{f} g\right)(\alpha)-f(\alpha)(T g)(\alpha) \\
& =\left(T M_{f} g\right)(\alpha)-\left(M_{f} T g\right)(\alpha)
\end{aligned}
$$

Since $J$ is not a Blaschke sequence, this means $T M_{f} g=M_{f} T g$. Therefore $T M_{f}=M_{f} T$.

Lemma 3.7 (see [13]). Suppose $f: G \rightarrow \Omega$ is a surjective analytic function and for each $\xi \in \Omega$, $n(\xi)$ is the number of points in $f^{-1}(\xi)$. Then

$$
\int_{G}\left|f^{\prime}\right|^{2} d A=\int_{\Omega} n(\xi) d A(\xi)
$$

Proposition 3.8. Let $f$ be in $R(\mathbb{D})$ and $f$ is analytic on $\overline{\mathbb{D}}$. Suppose for each $\xi \in f(\mathbb{D})$, there are $n$ points in $f^{-1}(\xi)$. Then for $g \in R(f(\mathbb{D}))$, we have $\mathcal{A}^{\prime}\left(M_{f}\right) \subset \mathcal{A}^{\prime}\left(M_{g \circ f}\right)$.

Proof. By the Embedding Theory of Sobolev space, $g \circ f \in C(\overline{\mathbb{D}})$. Therefore

$$
\int_{\mathbb{D}}|g(f(z))|^{2} d A(z) \leq \pi\|g\|_{\infty}
$$

By Lemma 3.7,

$$
\begin{aligned}
\int_{\mathbb{D}}\left|[g(f(z))]^{\prime}\right|^{2} d A(z) & =\int_{\mathbb{D}}\left|g^{\prime}(f(z))\right|^{2}\left|f^{\prime}(z)\right|^{2} d A(z) \\
& =n \int_{f(\mathbb{D})}\left|g^{\prime}(\omega)\right|^{2} d A(\omega) \\
& \leq n\|g\|_{R(f(\mathbb{D}))} .
\end{aligned}
$$

Since $f$ is analytic on $\mathbb{D}, f^{\prime}(z), f^{\prime \prime}(z)$ is bounded on $\mathbb{D}$. Hence

$$
\begin{gathered}
\int_{\mathbb{D}}\left|g^{\prime \prime}(f(z))\right|^{2}\left|f^{\prime}(z)\right|^{4} d A(z) \\
\leq\left\|f^{\prime}(z)\right\|_{\infty} \int_{\mathbb{D}}\left|g^{\prime \prime}(f(z))\right|^{2}\left|f^{\prime}(z)\right|^{2} d A(z) \\
\leq n\left\|f^{\prime}(z)\right\|_{\infty}\|g\|_{R(f(\mathbb{D}))^{\prime}} \\
\int_{\mathbb{D}}\left|g^{\prime}(f(z))\right|^{2}\left|f^{\prime \prime}(z)\right|^{2} d A(z) \leq\left\|f^{\prime \prime}(z)\right\|_{\infty}\|g\|_{R(f(\mathbb{D}))^{\prime}}
\end{gathered}
$$


Therefore,

$$
\int_{\mathbb{D}}\left|[g(f(z))]^{\prime \prime}\right|^{2} d A(z)<\infty
$$

By (3.24), (3.25), and (3.27), we have $g \circ f \in R(\mathbb{D})$.

For all $\beta \in f(\mathbb{D})$, because $((g(z)-g(\beta)) /(z-\beta)) \in R(f(\mathbb{D})),((g \circ f(z)-\beta) /(f(z)-\beta)) \in$ $R(\mathbb{D})$. For all $\alpha \in \mathbb{D}$

$$
g(f(z))-g(f(\alpha))=\frac{g(f(z))-g(f(\alpha))}{f(z)-f(\alpha)}(f(z)-f(\alpha)),
$$

so that

$$
(g(f(\mathrm{z}))-g(f(\alpha))) R(\mathbb{D}) \subset(f(z)-f(\alpha)) R(\mathbb{D}) .
$$

Set $T \in \mathscr{A}^{\prime}\left(M_{f}\right)$. By Lemma 3.6, for all $\alpha \in \mathbb{D}, T^{*} k_{\alpha} \perp(f-f(\alpha)) R(\mathbb{D})$. Hence, $T^{*} k_{\alpha} \perp$ $(g(f(z))-g(f(\alpha))) R(\mathbb{D})$ and we have $T \in \mathcal{A}^{\prime}\left(M_{g \circ f}\right)$.

Corollary 3.9. If $B_{n}(z)=\prod_{i=1}^{n}\left(z-a_{i}\right) /\left(1-\overline{a_{i}} z\right),\left(a_{i} \neq a_{j}, i \neq j,\left|a_{i}\right|<1\right)$ and $f \in R(\mathbb{D}), \mathscr{A}^{\prime}\left(M_{B_{n}}\right) \subset$ $A^{\prime}\left(M_{f \circ B_{n}}\right)$.

Proposition 3.10. Let $f$ be in $R(\mathbb{D})$ and $B_{n}$ is an $n$-Blaschke product. If $\mathscr{A}^{\prime}\left(M_{f}\right)=\mathscr{A}^{\prime}\left(M_{z}\right)=$ $\left\{M_{g} ; g \in R(\mathbb{D})\right\}$, then $\mathscr{A}^{\prime}\left(M_{f \circ B_{n}}\right)=\mathscr{A}^{\prime}\left(M_{B_{n}}\right)$.

Proof. From [3], we know that $\mathscr{A}^{\prime}\left(M_{z}\right)=\left\{M_{g} ; g \in R(\mathbb{D})\right\}$. By Lemma 3.3, $M_{B_{n}} \sim \oplus_{1}^{n} M_{z}$. Then there exists an invertible operator $X$ in $\mathcal{L}\left(R(\mathbb{D}), \oplus_{1}^{n} R(\mathbb{D})\right)$ such that $X M_{B_{n}} X^{-1}=\oplus_{1}^{n} M_{z}$. Since $M_{f \circ B_{n}}=f\left(M_{B_{n}}\right)$, we have $X M_{f \circ B_{n}} X^{-1}=\oplus_{1}^{n} M_{f}$. Therefore, we will only prove that $\mathcal{A}^{\prime}\left(\oplus_{1}^{n} M_{f}\right)=\mathcal{A}^{\prime}\left(\oplus_{1}^{n} M_{z}\right)$. Set $T \in \mathcal{A}^{\prime}\left(\oplus_{1}^{n} M_{f}\right)$ and

$$
T=\left(\begin{array}{cccc}
T_{11} & T_{12} & \cdots & T_{1 n} \\
T_{12} & T_{13} & \cdots & T_{2 n} \\
\vdots & \vdots & \cdots & \vdots
\end{array}\right) \begin{gathered}
R(\mathbb{D}) \\
\vdots
\end{gathered}
$$

Since

$$
\begin{aligned}
T\left(\oplus_{1}^{n} M_{f}\right) & =\left(\begin{array}{cccc}
T_{11} & T_{12} & \cdots & T_{1 n} \\
T_{12} & T_{13} & \cdots & T_{2 n} \\
\vdots & \vdots & \cdots & \vdots
\end{array}\right)\left(\begin{array}{ccc}
M_{f} & 0 & \cdots \\
0 & M_{f} & \cdots \\
0 & & \ddots
\end{array}\right) \\
& =\left(\oplus_{1}^{n} M_{f}\right) T=\left(\begin{array}{ccc}
M_{f} & 0 & \cdots \\
0 & M_{f} & \cdots \\
0 & & \ddots
\end{array}\right)\left(\begin{array}{cccc}
T_{11} & T_{12} & \cdots & T_{1 n} \\
T_{12} & T_{13} & \cdots & T_{2 n} \\
\vdots & \vdots & \cdots & \vdots
\end{array}\right),
\end{aligned}
$$

we have $T_{i j} M_{f}=M_{f} T_{i j}$ for $i, j=1,2, \ldots$ So $T_{i j} \in \mathscr{A}^{\prime}\left(M_{f}\right)=\mathscr{A}^{\prime}\left(M_{z}\right)$ and $\mathscr{A}^{\prime}\left(\oplus_{1}^{n} M_{f}\right) \subset$ $\mathscr{A}^{\prime}\left(\oplus_{1}^{n} M_{z}\right)$. Similarly, $\mathscr{A}^{\prime}\left(\oplus_{1}^{n} M_{z}\right)=\mathscr{A}^{\prime}\left(\oplus_{1}^{n} M_{f}\right)$. Hence $\mathscr{A}^{\prime}\left(M_{f \circ B_{n}}\right)=\mathscr{A}^{\prime}\left(M_{B_{n}}\right)$. 
Let $f$ be an injective function in $R(\mathbb{D})$ and $\Omega=f(\mathbb{D})$. Then for each $z_{0} \in \mathbb{D}$, it is obvious that $f\left(z_{0}\right)$ is not in $f(\mathbb{T})$. Denote the component of $\rho_{s-F}\left(M_{f}\right)$ containing $f\left(z_{0}\right)$ as $\Omega$, then $z_{0}$ is the only zero point of $f(z)-f\left(z_{0}\right)$ in $\mathbb{D}$. By Lemma 2.2, $M_{f}^{*}$ is a Cowen-Douglas operator with index 1. By Lemma 2.5, we have $\mathcal{A}^{\prime}\left(M_{f}\right)=\left\{M_{f} ; f \in R(\mathbb{D})\right\}=\mathscr{A}^{\prime}\left(M_{z}\right)$. So the following corollary is obtained.

Corollary 3.11. Let $f$ be a univalent analytic function in $R(\mathbb{D})$ and $B_{n}$ be an $n$-Blaschke product. Then $\mathcal{A}^{\prime}\left(M_{B_{n}}\right)=\mathscr{A}^{\prime}\left(M_{f \circ B_{n}}\right)$.

Lemma 3.6 shows if $f$ is in $R(\mathbb{D})$ and $z_{0}$ is in $\mathbb{D}$, then

$$
\bigvee\left\{T^{*} k_{z_{0}}: T \in \mathcal{A}^{\prime}\left(M_{f}\right)\right\} \subset\left[\left(f-f\left(z_{0}\right)\right) R(\mathbb{D})\right]^{\perp}=\operatorname{ker} M_{f-f\left(z_{0}\right)}^{*}
$$

Easy examples show that ker $M_{f-f\left(z_{0}\right)}^{*} \subset \bigvee\left\{T^{*} k_{z_{0}}: T \in \mathcal{A}^{\prime}\left(M_{f}\right)\right\}$ is not true. The following proposition shows that if $f(z)=z^{n}, \bigvee\left\{T^{*} k_{z_{0}}: T \in \mathcal{A}^{\prime}\left(M_{f}\right)\right\}=\operatorname{ker} M_{f-f\left(z_{0}\right)}^{*}$.

Lemma 3.12 (see $[1]$ ). Let $f \in R(\mathbb{D}), M_{f}^{*} \in \mathbb{B}_{n}(\Omega), z_{0} \in D_{1}:=f^{-1}(\Omega)$, and

$$
f(z)-f\left(z_{0}\right)=\left(z-z_{0}\right)^{h_{1}}\left(z-z_{1}\right)^{h_{2}} \cdots\left(z-z_{l}\right)^{h_{l+1}} g_{z_{0}}(z)
$$

where $\left\{z_{i}\right\}_{i=1}^{l} \subset D_{1}$ are pairwise distinct, $\sum_{i=1}^{l+1} h_{i}=n, g_{z_{0}}(z) \neq 0, z \in \overline{\mathbb{D}}$. Choose $k_{z_{i}}^{1}, \ldots, k_{z_{i}}^{h_{i+1}-1} \in$ $R(\mathbb{D})$ such that

$$
M_{z-z_{i}}^{*} k_{z_{i}}^{1}=k_{z_{i}}, \ldots, M_{z-z_{i}}^{*} k_{z_{i}}^{h_{i+1}-1}=k_{z_{i}}^{h_{i+1}-2}, \quad(0 \leq i \leq l) .
$$

Then there exists a linearly independent set

$$
K_{z_{0}}:=\left\{k_{z_{0}}, k_{z_{0}}^{1}, \ldots, k_{z_{0}}^{h_{1}-1}, k_{z_{1}}, \ldots, k_{z_{1}}^{h_{2}-1}, \ldots, k_{z_{l}}^{h_{l+1}-1}\right\}
$$

such that $\operatorname{ker} M_{f-f\left(z_{0}\right)}^{*}=\bigvee K_{z_{0}}$.

Let $n \geq 2$ and $\omega$ be the $n$th root of 1 , that is, $\omega \in \mathbb{C}$ and $\omega^{n}=1$. Let $\Delta_{n}$ denote the Vandermonde determinant of order $n$ :

$$
\Delta_{n}=\left|\begin{array}{ccccc}
1 & 1 & 1 & \cdots & 1 \\
1 & \omega & \omega^{2} & \cdots & \omega^{n-1} \\
\vdots & \vdots & \vdots & & \vdots \\
1 & \omega^{n-1} & \omega^{2(n-1)} & \cdots & \omega^{(n-1)^{2}}
\end{array}\right|
$$

For $1 \leq i, j \leq n$, the $(i, j)$-cofactor will be denoted by $\Delta_{i j}$. 
Lemma 3.13 (see [1]). $A \in \mathcal{A}^{\prime}\left(M_{z^{n}}\right)$ if and only if for all $g \in R(\mathbb{D})$ and $z \neq 0$,

$$
(A g)(z)=\sum_{i=1}^{n} \alpha_{i}(z) g\left(\omega^{i-1} z\right)
$$

where $\alpha_{i}(z)=\left(\sum_{j=1}^{n} \Delta_{i j}\left(h_{j} / z^{j-1}\right)\right) / \Delta_{n}$, for some $\left\{h_{j}\right\}_{j=1}^{n}$ in $R(\mathbb{D})$.

Proposition 3.14. For all $z_{0} \in \mathbb{D}, \bigvee\left\{T^{*} k_{z_{0}}: T \in \mathscr{A}^{\prime}\left(M_{z^{n}}\right)\right\}=\left[\left(z^{n}-z_{0}^{n}\right) R(\mathbb{D})\right]^{\perp}$.

Proof. By Lemma 3.6, $\bigvee\left\{T^{*} k_{z_{0}}: T \in \mathcal{A}^{\prime}\left(M_{z^{n}}\right)\right\} \subset\left[\left(z^{n}-z_{0}^{n}\right) R(\mathbb{D})\right]^{\perp}$. Now we prove that $\left[\left(z^{n}-\right.\right.$ $\left.\left.z_{0}^{n}\right) R(\mathbb{D})\right]^{\perp} \subset \bigvee\left\{T^{*} k_{z_{0}}: T \in \mathscr{A}^{\prime}\left(M_{z^{n}}\right)\right\}$.

Since

$$
z^{n}-z_{0}^{n}=\left(z-z_{0}\right)\left(z-\omega z_{0}\right) \cdots\left(z-\omega^{n-1} z_{0}\right)
$$

by Lemma 3.12,

$$
\left[\left(z^{n}-z_{0}^{n}\right) R(\mathbb{D})\right]^{\perp}=\operatorname{ker} M_{z^{n}-z_{0}^{n}}^{*}=\bigvee\left\{k_{z_{0}}, k_{\omega z_{0}}, \ldots, k_{\omega^{n-1} z_{0}}\right\}
$$

Set $f=a_{1} k_{z_{0}}+a_{2} k_{\omega z_{0}}+\cdots+a_{n} k_{\omega^{n-1} z_{0}} \in \operatorname{ker} M_{z^{n}-z_{0}^{n}}^{*}$ with $a_{1}, a_{2}, \ldots, a_{n} \in \mathbb{C}$. For all $g \in R(\mathbb{D})$, we define an operator $T: R(\mathbb{D}) \mapsto R(\mathbb{D})$ as follows:

$$
T g(z)=\overline{a_{1}} g(z)+\overline{a_{2}} g(\omega z)+\cdots+\overline{a_{n}} g\left(\omega^{n-1} z\right)
$$

By Lemma 3.13, $T \in \mathscr{A}^{\prime}\left(M_{z^{n}}\right)$. For all $\lambda \in \mathbb{D}$,

$$
\begin{aligned}
T^{*} k_{z_{0}}(\lambda) & =\left\langle T^{*} k_{z_{0}}, k_{\curlywedge}\right\rangle=\left\langle k_{z_{0}}, T k_{\curlywedge}\right\rangle \\
& =\left\langle k_{z_{0}}, \overline{a_{1}} k_{\curlywedge}(z)+\overline{a_{2}} k_{\curlywedge}(\omega z)+\cdots+\overline{a_{n}} k_{\curlywedge}\left(\omega^{n-1} z\right)\right\rangle \\
& =a_{1} \overline{\left\langle k_{\lambda}(z), k_{z_{0}}\right\rangle}+a_{2} \overline{\left\langle k_{\lambda}(\omega z), k_{z_{0}}\right\rangle}+\cdots+a_{n} \overline{\left\langle k_{\lambda}\left(\omega^{n-1} z\right), k_{z_{0}}\right\rangle} \\
& =a_{1} \overline{k_{\lambda}\left(z_{0}\right)}+a_{2} \overline{k_{\curlywedge}\left(\omega z_{0}\right)}+\cdots+a_{n} \overline{k_{\curlywedge}\left(\omega^{n-1} z_{0}\right)} .
\end{aligned}
$$

For $0 \leq i \leq n-1$,

$$
\begin{aligned}
\overline{k_{\curlywedge}\left(\omega^{i} z_{0}\right)} & =\sum_{m=0}^{\infty} \overline{\beta_{m}^{2}(\bar{\lambda})^{m}\left(\omega^{i} z_{0}\right)^{m}} \\
& =\sum_{m=0}^{\infty} \beta_{m}^{2}\left(\overline{\omega^{i} z_{0}}\right)^{m} \lambda^{m} \\
& =k_{\omega^{i} z_{0}}(\lambda) .
\end{aligned}
$$


Therefore,

$$
T^{*} k_{z_{0}}(\lambda)=a_{1} k_{z_{0}}(\lambda)+a_{2} k_{\omega z_{0}}(\lambda)+\cdots+a_{n} k_{\omega^{n-1} z_{0}}(\lambda)=f(\lambda),
$$

that is $T^{*} k_{z_{0}}=f$. Then $f \in \bigvee\left\{T^{*} k_{z_{0}}: T \in \mathcal{A}^{\prime}\left(M_{z^{n}}\right)\right\}$ and we have $\left[\left(z^{n}-z_{0}^{n}\right) R(\mathbb{D})\right]^{\perp} \subset \bigvee\left\{T^{*} k_{z_{0}}\right.$ : $\left.T \in \mathcal{A}^{\prime}\left(M_{z^{n}}\right)\right\}$.

Easy examples show that, in general, the converse of Proposition 3.8 is false. But the following case is an exception. To prove it, we need the following lemma.

Lemma 3.15. Let $f=h\left(z^{n}\right)$ be in $R(\mathbb{D})$ and $h$, analytic on $\mathbb{D}$. Then $h$ is in $R(\mathbb{D})$.

Proof. Since $h$ is analytic on $\mathbb{D}$, we have $h(z)=\sum_{m=0}^{\infty} h_{m} z^{m}$, hence,

$$
f(z)=h\left(z^{n}\right)=\sum_{m=0}^{\infty} h_{m} z^{n m}
$$

From $f$ being in $R(\mathbb{D})$, we have

$$
\sum_{m=0}^{\infty} \frac{\left|h_{m}\right|^{2}}{\beta_{n m}^{2}}<+\infty
$$

Because $\left\{\beta_{n}\right\}$ is monotonically decreasing, $\beta_{m} \geq \beta_{n m}$ for all $m \geq 0$. So

$$
\sum_{m=0}^{\infty} \frac{\left|h_{m}\right|^{2}}{\beta_{m}^{2}}<\sum_{m=0}^{\infty} \frac{\left|h_{m}\right|^{2}}{\beta_{n m}^{2}}<+\infty
$$

and this shows that $h$ is in $R(\mathbb{D})$.

Proposition 3.16. If $f \in R(\mathbb{D})$ and $\mathscr{A}^{\prime}\left(M_{z^{n}}\right) \subset \mathcal{A}^{\prime}\left(M_{f}\right)$, then there exists $h$ being in $R(\mathbb{D})$ such that $f=h\left(z^{n}\right)$.

Proof. By Proposition 3.14, for all $z_{0} \in \mathbb{D}$, we have

$$
\begin{aligned}
{\left[\left(z^{n}-z_{0}^{n}\right) R(\mathbb{D})\right]^{\perp} } & =\bigvee\left\{T^{*} k_{z_{0}}: T \in \mathscr{A}^{\prime}\left(M_{z^{n}}\right)\right\} \\
& \subset \bigvee\left\{T^{*} k_{z_{0}}: T \in \mathcal{A}^{\prime}\left(M_{f}\right)\right\} \\
& \subset\left[\left(f-f\left(z_{0}\right)\right) R(\mathbb{D})\right]^{\perp} .
\end{aligned}
$$

For each $\lambda \in \mathbb{D}$, we can find $z_{0} \in \mathbb{D}$ such that $z_{0}^{n}=\lambda$. We define $h$ on $\mathbb{D}$ by $h(\lambda)=f\left(z_{0}\right)$ and $h$ is well defined. Indeed, set $z_{0}^{n}=z_{1}^{n}=\lambda$. Then

$$
k_{z_{1}} \in\left[\left(z^{n}-z_{0}^{n}\right) R(\mathbb{D})\right]^{\perp} \subset\left[\left(f-f\left(z_{0}\right)\right) R(\mathbb{D})\right]^{\perp} .
$$

Hence $f\left(z_{1}\right)=f\left(z_{0}\right)$. 
For $0 \neq \lambda_{0} \in \mathbb{D}$, we have $z_{0} \neq 0$. Therefore,

$$
\begin{aligned}
\lim _{\lambda \rightarrow \lambda_{0}} \frac{h(\lambda)-h\left(\lambda_{0}\right)}{\lambda-\lambda_{0}} & =\lim _{z \rightarrow z_{0}} \frac{f(z)-f\left(z_{0}\right)}{z^{n}-z_{0}^{n}} \\
& =\lim _{z \rightarrow z_{0}} \frac{f(z)-f\left(z_{0}\right)}{z-z_{0}} \frac{z-z_{0}}{z^{n}-z_{0}^{n}} \\
& =\frac{f^{\prime}\left(z_{0}\right)}{n z_{0}^{n-1}} .
\end{aligned}
$$

If $\lambda=0, z_{0}=0$. Since

$$
z^{n} R(\mathbb{D}) \supset(f-f(0)) R(\mathbb{D})
$$

there exists $g \in R(\mathbb{D})$ such that $f-f(0)=z^{n} g$. Hence,

$$
\begin{aligned}
\lim _{\lambda \rightarrow 0} \frac{h(\lambda)-h(0)}{\lambda-0} & =\lim _{z \rightarrow 0} \frac{f(z)-f(0)}{z^{n}} \\
& =\lim _{z \rightarrow 0} \frac{z^{n} g(z)}{z^{n}} \\
& =g(0) .
\end{aligned}
$$

So $h$ is analytic on $\mathbb{D}$. By Lemma 3.15, we have $h \in R(\mathbb{D})$ and $f(z)=h\left(z^{n}\right)$. a. Define

For each $f \in R(\mathbb{D})$ and $a \notin f(\partial \mathbb{D}), \eta(f(\partial \mathbb{D}), a)$ denote the winding number of $f(\partial \mathbb{D})$ at

$$
s=k(f)=\inf \{\eta(f(\partial \mathbb{D}), a): \eta(f(\partial \mathbb{D}), a) \neq 0\} .
$$

Proposition 3.17. If $f \in R(\mathbb{D})$ is a nonconstant entire function and $s=k(f)$, then $\mathscr{A}^{\prime}\left(M_{f}\right)=$ $A^{\prime}\left(M_{z^{s}}\right)$.

Proof. By Theorem 1 in [14], there exists an entire function $h$ such that $f(z)=h\left(z^{S}\right)$ and $k(h)=$ 1. Since $h$ is an entire function, $h, h^{\prime}$, and $h^{\prime \prime}$ are all bounded and analytic on $\overline{\mathbb{D}}$. So $h \in R(\mathbb{D})$. By $k(h)=1$, there is only one zero of $h-\lambda$ in $\mathbb{D}$ for some $\lambda$. By Lemma 2.2, $M_{h}^{*} \in B_{1}(\Omega)$. By Proposition 3.10, $\mathscr{A}^{\prime}\left(M_{f}\right)=\mathcal{A}^{\prime}\left(M_{h\left(z^{s}\right)}\right)=\mathscr{A}^{\prime}\left(M_{z^{s}}\right)$.

\section{Acknowledgment}

The research is supported by NSFC 11001078.

\section{References}

[1] Z. Wang and Y. Liu, "Multiplication operators on Sobolev disk algebra," Science in China Series A, vol. 48, no. 10, pp. 1395-1410, 2005. 
[2] C. L. Jiang and Z. Y. Wang, Structure of Hilbert Spaces Operators, World Scientific, Singapore, 2006.

[3] Y. Liu and Z. Wang, "The commutant of the multiplication operators on Sobolev disk algebra," Journal of Analysis and Applications, vol. 2, no. 2, pp. 65-86, 2004.

[4] D. A. Herrero, "Spectral pictures of operators in the Cowen-Douglas class $B_{n}(\Omega)$ and its closure," Journal of Operator Theory, vol. 18, no. 2, pp. 213-222, 1987.

[5] D. A. Herrero, Approximation of Hilbert Space Operatrs I, Research Notes in Mathematics 224, Longman, Essex, UK, 2nd edition, 1989.

[6] J. B. Conway, Course in Functional Analysis, vol. 96 of Graduate Texts in Mathematics, Springer, New York, NY, USA, 2nd edition, 1990.

[7] Z. Wang, R. Zhao, and Y. Jin, "Finite Blaschke product and the multiplication operators on Sobolev disk algebra," Science in China Series A, vol. 52, no. 1, pp. 142-146, 2009.

[8] C. Jiang and Z. Wang, Strongly Irreducible Operators on Hilbert Space, vol. 389 of Pitman Research Notes in Mathematics Series, Longman, Harlow, UK, 1998.

[9] F. Gilfeather, "Strong reducibility of operators," Indiana University Mathematics Journal, vol. 22, pp. 393-397, 1972-1973.

[10] Y.-Q. Liu and Z.-Y. Wang, "Invariant subspaces of Sobolev disk algebra," Journal of Mathematical Research and Exposition, vol. 26, no. 2, pp. 233-238, 2006.

[11] J. B. Conway, Functions of One Complex Variable I, vol. 11 of Graduate Texts in Mathematics, Springer, New York, NY, USA, 2nd edition, 1978.

[12] J. A. Deddens and T. K. Wong, "The commutant of analytic Toeplitz operators," Transactions of the American Mathematical Society, vol. 184, pp. 261-273, 1973.

[13] J. B. Conway, Functions of One Complex Variable. II, vol. 159 of Graduate Texts in Mathematics, Springer, New York, NY, USA, 1995.

[14] I. N. Baker, J. A. Deddens, and J. L. Ullman, "A theorem on entire functions with applications to Toeplitz operators," Duke Mathematical Journal, vol. 41, pp. 739-745, 1974. 


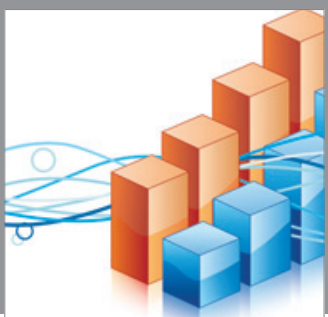

Advances in

Operations Research

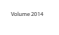

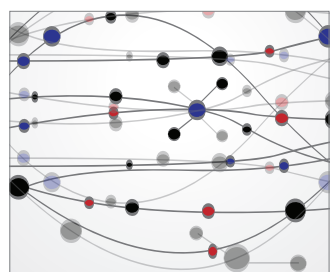

\section{The Scientific} World Journal
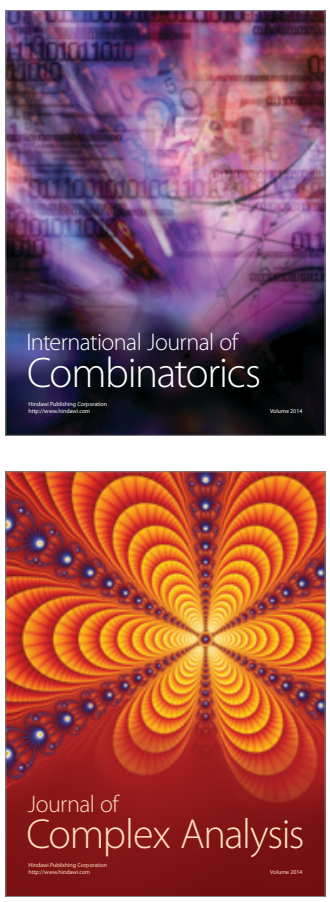

International Journal of

Mathematics and

Mathematical

Sciences
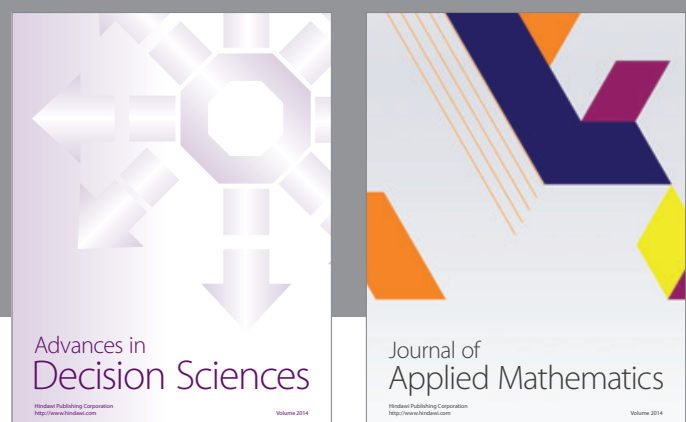

Journal of

Applied Mathematics
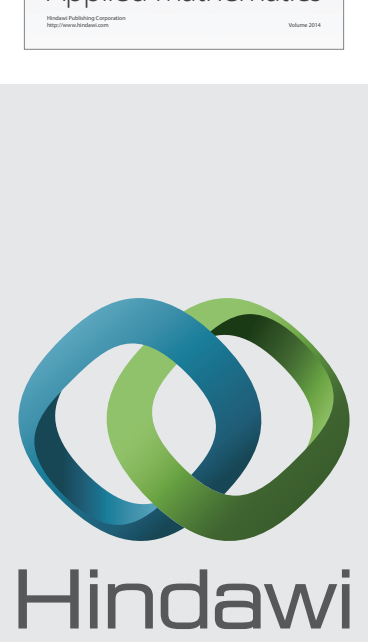

Submit your manuscripts at http://www.hindawi.com
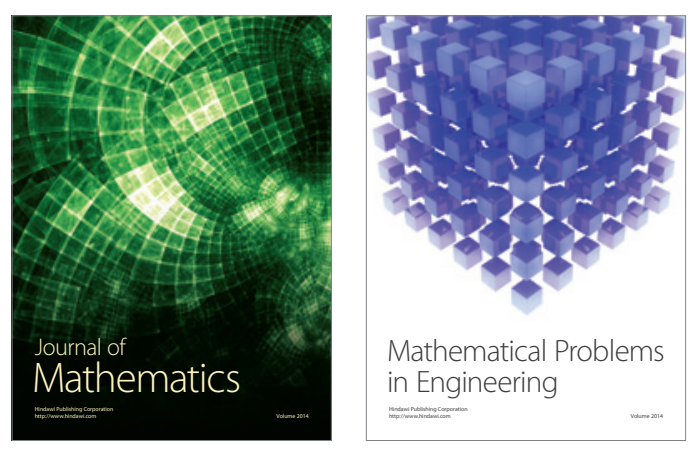

Mathematical Problems in Engineering
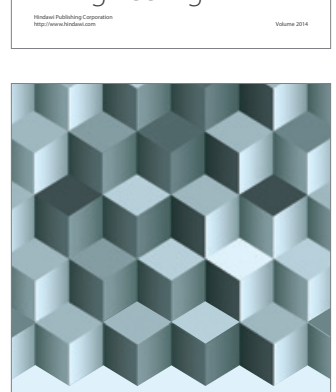

Journal of

Function Spaces
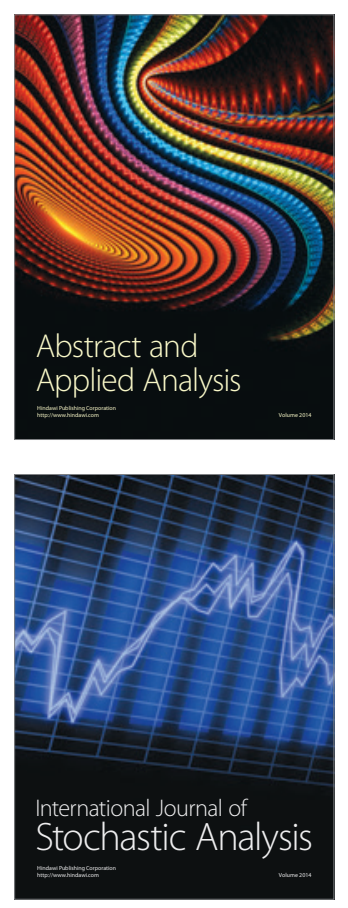

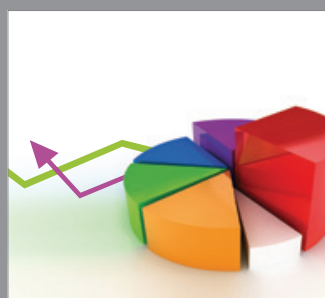

ournal of

Probability and Statistics

Promensencen
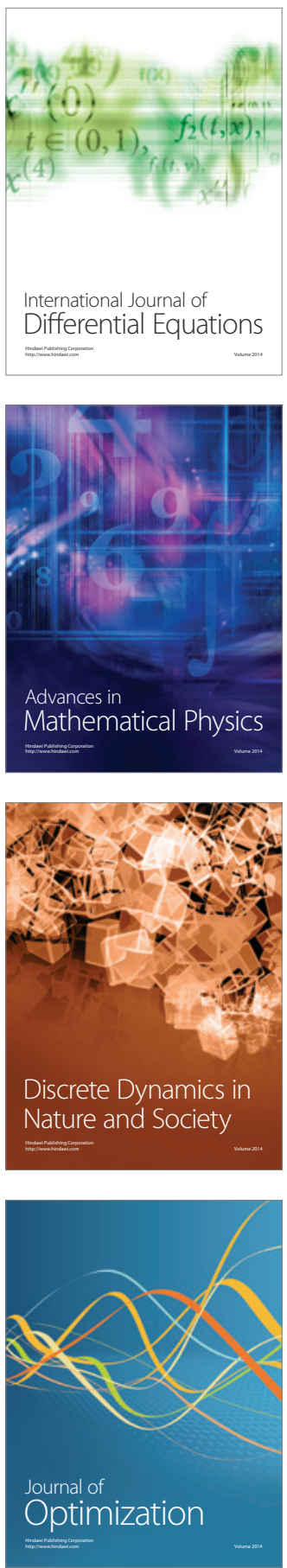